\title{
Evaluation of Network Parameters of a Sensor Node Deployment Strategy in Wireless Sensor Network for Hilly Terrains
}

\author{
Sunita Saha \\ Department of Computer Science and Engineering, NERIST, Nirjuli, 791109, India \\ E-mail: saha.sunita92@gmail.com
}

Received: 28 September 2018; Accepted: 18 November 2018; Published: 08 January 2019

\begin{abstract}
The localization of sensor nodes in a Wireless Sensor Network (WSN) can be examined by the resultant network parameters of covered sensing area and superimposed area. The measurement of covered sensing area is out of the total surface area how much geographical area can be sensed by the placed sensors and superimpose area is out of the total coverage area how much area is sensed or covered by more than one sensor node. A Wireless Sensor Network can be claimed to be productive only if it produces a good degree of coverage area with respect to less superimposing area and with the use of minimum sensor count also a degree of connectivity. To ensure the performance it is important to place the sensor nodes in a Wireless Sensor Network in its appropriate location. The placement of sensor nodes in 3D Wireless Sensor Network deals with complex mathematical modeling and higher sensor count compared to 2D Wireless Sensor Network. In this paper computation of actual covered area and superimposing area are highlighted after designing a network with a particular node placement method for Hilly Surfaces.
\end{abstract}

Index Terms-Localization, Terrains, Superimposed area, covered sensing area, Sensor count, Wireless Sensor Network.

\section{INTRODUCTION}

Wireless Sensor Networks consist of large number of tiny, inexpensive, low power and resources constrained devices called Sensor Nodes (SN) whose purpose is to sense or monitor changes in critical parameters such as sound, environmental pressure and temperature and communicate the observations locally or to the Base Station (BS) in single or multi-hop routes. WSNs play a vital role in many applications such as health care, environmental monitoring, military surveillance etc. WSNs are prone to adversarial attacks due to a limited amount of memory embedded on Sensor Nodes, lack of fixed infrastructure and uncontrolled environment. Security requirements are mandatory since WSNs are often deprived of human support to maintain secure communication between sensor nodes. WSNs and ad-hoc networks have similar security requirements.

The concern about Coverage, Connectivity and Lifetime together into one simple parameter would help much in evaluation of performance of a designed network So, Coverage and Connectivity can be considered as basic requirements in a Wireless Sensor Network.

In a WSN when the height of the sensor nodes positions' is not negligible as compared to the breadth and length of the deployment field it is categorized as three-dimensional (3D). To find an optimal way to localize the sensor nodes on the targeted 3D WSN is the major issue to get the desired degree of coverage and a reliable connectivity on for the chosen field. The solution should yield a lower bound on the number of nodes needed to achieve full coverage with minimum superposition. However, optimization of network parameters such as coverage and superposition in 3D WSNs deals with complex mathematics and its intrinsic design challenges. 3D WSNs are not only difficult to visualize but their analysis is also computationally intensive.

\section{RELATED WORKS}

Numbers of sources are used to provide the general background information on this topic. In this section, a brief review of node deployment methods which solves Coverage and superimpose problem in Wireless Sensor Networks and techniques that are used to increase the lifetime using Coverage are discussed.

Sema Oktug and et al, their study went on 3D coverage under the heterogeneous environments for the newly proposed deployment strategies. The answers to questions like how many sensors are needed to cover a specified 3D terrain at a specified coverage percentage were evaluated. Simulation results demonstrate that depending on application requirements an appropriate deployment strategy could be selected to obtain required 3D coverage. [17]

Hassan Chizari and et al, they proposed a Delaunay Triangulation based method to place the sensor nodes on the targeted field which satisfied the value for all coverage measurement tools. The evaluated results 
proved that the proposed triangulation method achieved accurate coverage information, and provided many tools to compare QoC between different scenarios. ${ }^{[8,9]}$

Miao Jin and et $a l$, in ${ }^{[13]}$ they tackled the problem of optimal sensor deployment on 3D surfaces, aiming to achieve the highest overall sensing quality. The extensive simulations were conducted on various 3D mountain surface models to demonstrate the effectiveness of the proposed algorithms.

Usman Mansoor and Habib M. Ammari, they summarized major work conducted in the domain of coverage and connectivity in 3D WSNs. The evaluation of the node deployment scheme was shown with five regular space filling polyhedrons: cube, dodecahedron, icosahedron, octahedron, and tetrahedron. $\left.{ }^{[20}\right]$

Renan Nespolo and et al proposed a new method for node deployment in a 3D surface which is based on Delaunay triangulation. To obtain maximum coverage area using a minimum number of sensor nodes in threedimensional surfaces the deployment was performed

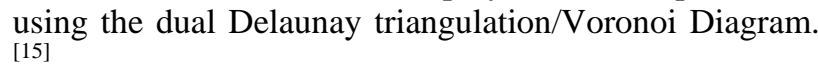

S. Surekha and et al, they used Delaunay Triangulation based deployment for the uncovered region in the WSN. In their work, area coverage problem was considered for irregularly shaped target fields. The Random deployment and Grid-based deployment of sensor nodes was analyzed and compared according to the results obtained. ${ }^{[18]}$

The novelty of the review lies in the different study angles and providing a comprehensive understanding of the coverage and connectivity ${ }^{[1,4,6,7]}$ in deployment phase $^{[2,3,10,14]}$ in WSNs.

In literature, the proposed methods considering threedimensional solutions showed relevant results for specific reliefs, indicating that it is possible to propose a flexible method to design a 3D WSN and to solve the problem of maximum coverage on that distinct three-dimensional surface. Based on the review, it may be revealed that computational geometry has a unique advantage on designing 3D Hilly Terrain of a surface and approximate the surface more smoothly which also provide an estimated environment for WSN and sensor node deployment on WSN. Also it has a great advantage on performance evaluation of WSN in terms of coverage of the network dealing with some of the polyhedrons. In this work, a new method is proposed where the design of the network is tried to be solved using geometric modeling as well as the performance of the network.

\section{METHODOLOGY}

In this project work, the main aim is to get higher efficiency for a network topology in terms of coverage and superimpose area with respect to sensor count which will increase the network performance and which is at the same time economical. The work can be divided into roughly five phases.

\section{A. Phases of the Proposed Method}

Phase1: Collection of surface data

Phase2: Generation of the simulated surface using Delaunay Triangulation

Phase3: Node deployment strategy

Phase4: Getting the sensor Count and position selection of the sensor nodes

Phase 5: Evaluation of coverage and superimpose Area.

\section{B. Sketch of the Proposed Node Placement Method}

The proposed sensor node placement method for Hilly Surfaces is represented in a flowchart as follow:

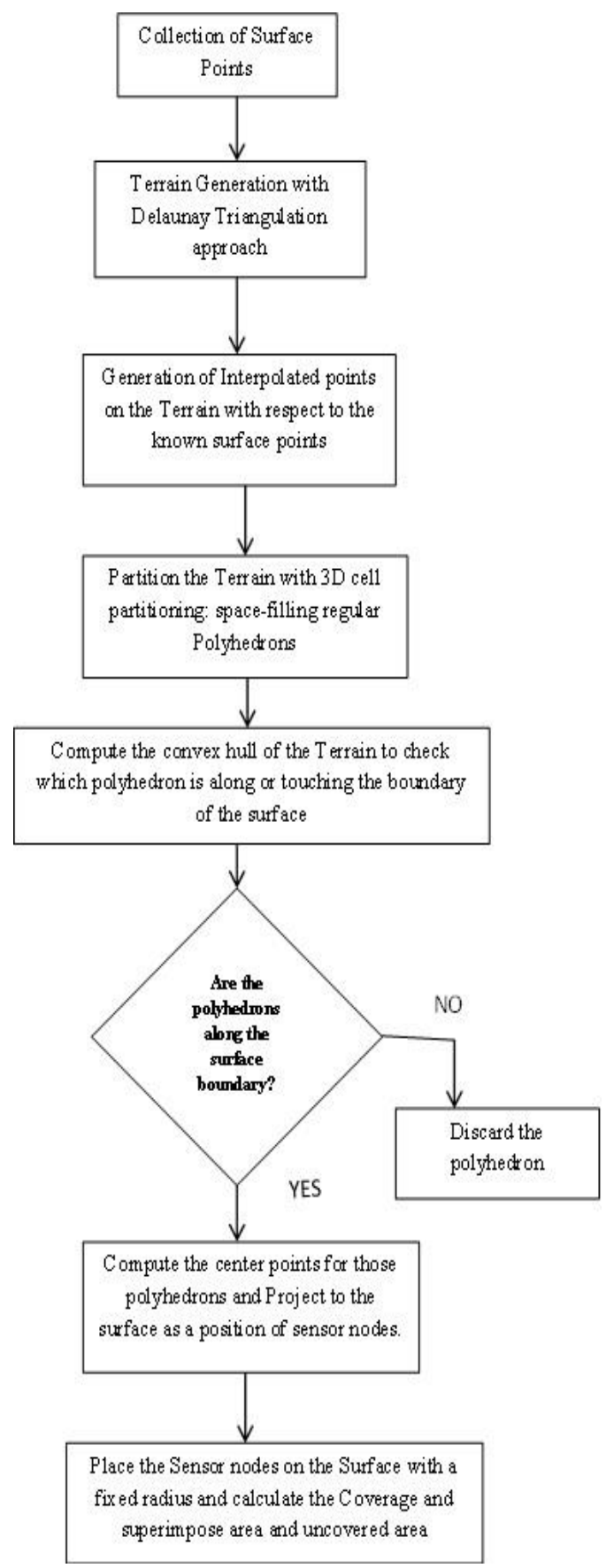

Fig.1. Flowchart of the node placement method 


\section{SURFACE GENERATION}

In this section, a hilly earth surface was chosen and made it a computer simulated environment to model the proposed designed approach and for the evaluation and of the model to check the efficiency. For the approximation of an Earth's surface, the traditional approach of terrain generation was followed with the Delaunay Triangulation approach to generate a terrain of the surface to work on it.

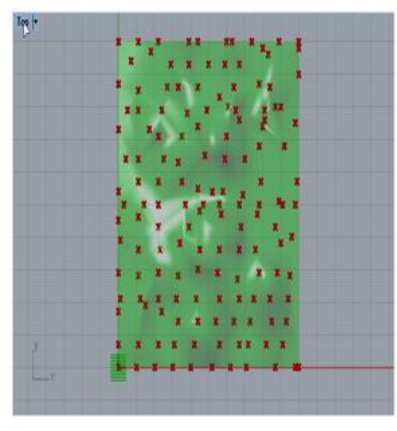

a

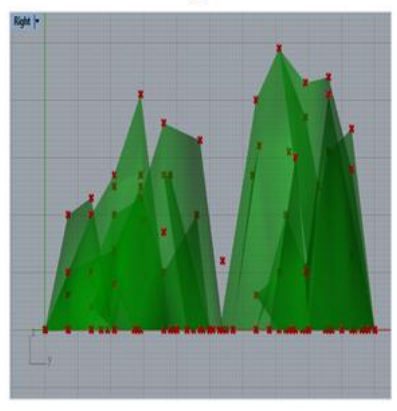

c

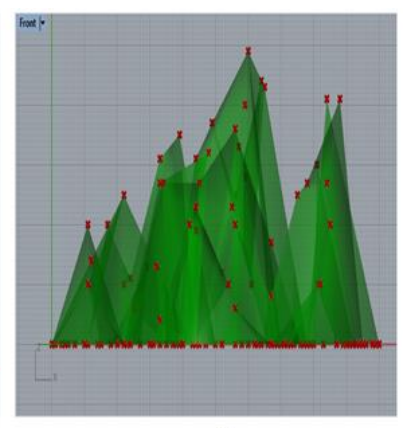

b

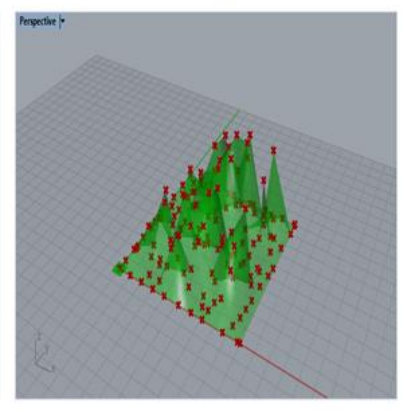

d
Fig.2. Simulated results for the surface generation with the surface points: (a) Top view, (b) Front view, (c) Right view, (d) Perspective view.

Total 2D Surface area, $T$ of the surface was calculated in $3 \mathrm{D}$ space for each triangulated face generated in the triangulation using coordinate geometry ${ }^{[19]}$.

$$
A=\frac{\overrightarrow{\left(P_{2}-P_{1}\right)} \times\left(\overrightarrow{\left.P_{3}-P_{1}\right)}\right.}{2}
$$

Here, $P_{1}, P_{2}, P_{3}$ are the surface points generating a triangulated face in the triangulation.

Total Surface area,

$$
T=A_{1}+A_{2}+A_{3}+\ldots \ldots . .+A_{n}
$$

Here $A_{1}, A_{2}, A_{3}, \ldots \ldots . ., A_{n}$ is the area of the each triangulated face generated in the triangulation.

\section{Sensor Placement Strategy}

Placement of sensor nodes in Wireless Sensor Network is one of the most challenging issues with a limited number of sensors in order to achieve maximum geographical coverage and connectivity among the sensor nodes on a targeted $3 \mathrm{D}$ field which dictates the overall network performance. There are various researches going on to improve the sensor placement strategy based on different algorithms.

\section{A. Partitioning of the Targeted Field}

In this work, a divide and conquer approach was considered to get the sensor count and projection method was used to localize the sensor nodes in the targeted field to get the maximum coverage. Volumetric quotient based approach was used to partition the space where the targeted surface resided. Space was divided by spacefilling polyhedron i.e. cube and centers of the cubes were considered as sensor count which were inside the surface boundary.

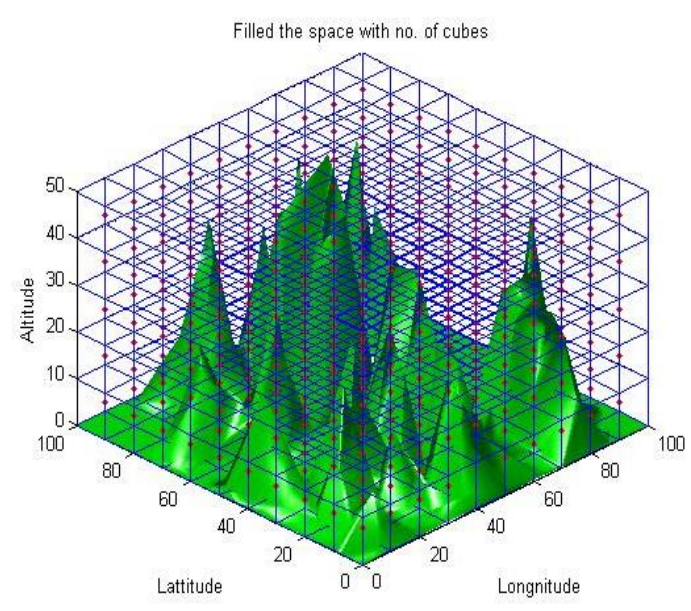

Fig.3. Simulated results for the partitioning of the surface with space filling polyhedrons: cube

\section{B. Localization of the Sensors on the Targeted Field}

To place the sensor nodes on the targeted field, the projection method was used to get the location of the sensor nodes. After getting the sensor count and the cube centers, the sensors positions were computed through projection method and nodes were placed on the field to get an efficient area coverage and connectivity. In this work, with the same number of sensor count, sensors were placed in two different positions as follows:

- Case 1: Project to the nearest known surface sample point as the sensor location.

- Case2: Project to the nearest surface point i.e. perpendicular projection. 


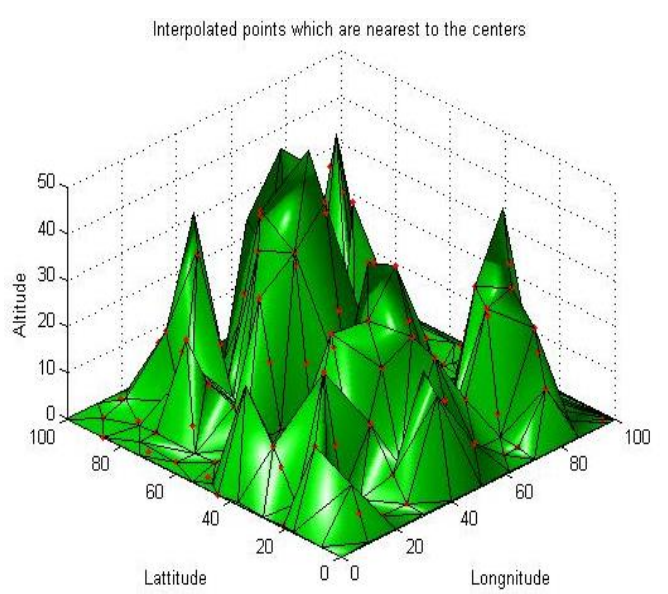

Fig.4. Sensor locations after projection to the terrain (Case1)

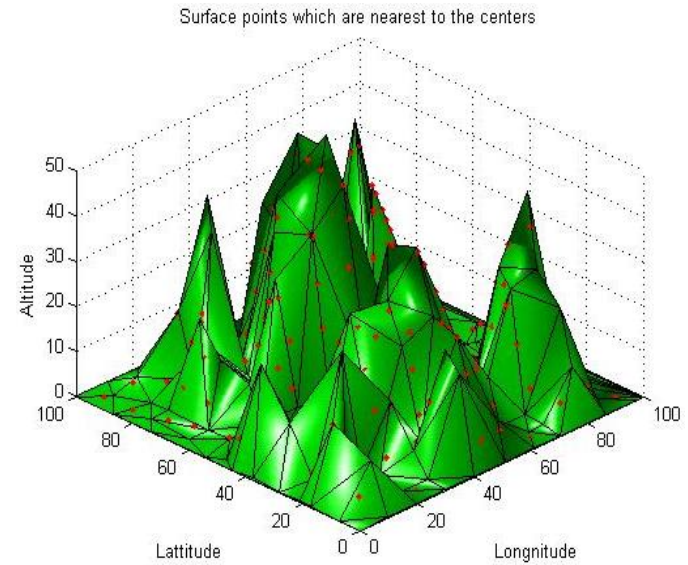

Fig.5. Sensor locations after projection to the terrain (Case2)

After projection of the sensors on the field, conflict locations of the sensors were discarded and considered only one sensor localization at a particular point with a fixed covering range.

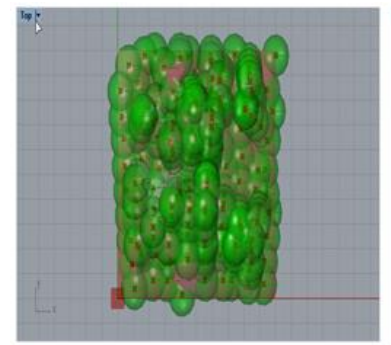

a

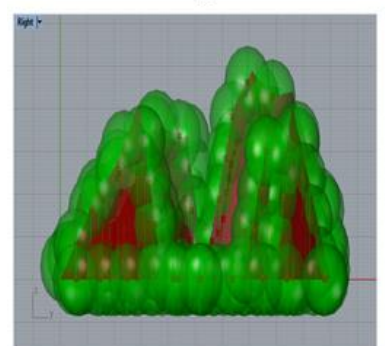

c

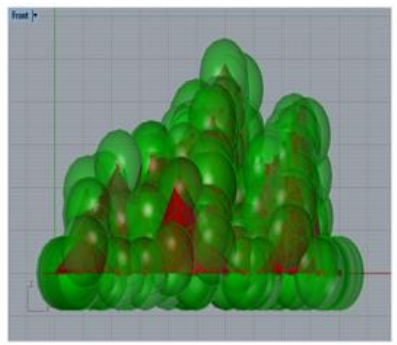

$\mathrm{b}$

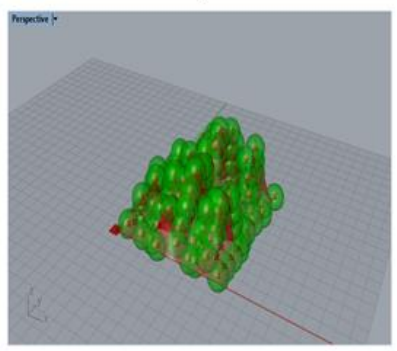

d
Fig.6. Simulated results for the Sensor node placement on the targeted field with a fixed covering range. (a) Top view, (b) Front view, (c) Right view, (d) Perspective view.

\section{COMPUTATION OF THE PARAMETERS}

After the placement of the sensor nodes on the targeted field, computation of coverage was done to check the efficiency of the placement method. In this work, geographical coverage was computed as follows:

\section{A. Coverage Calculation}

To get the actual geographical coverage with the placed sensor nodes, the intersection between the generated surface and the sensing covering spheres were evaluated until intersection area became null. Total coverage was calculated from the union of those intersection areas.

\section{Coverage $=$ Triangulated faces of the surface $\cap$ Sensor Nodes \\ Total Coverage Area $=\sum$ Coverage}
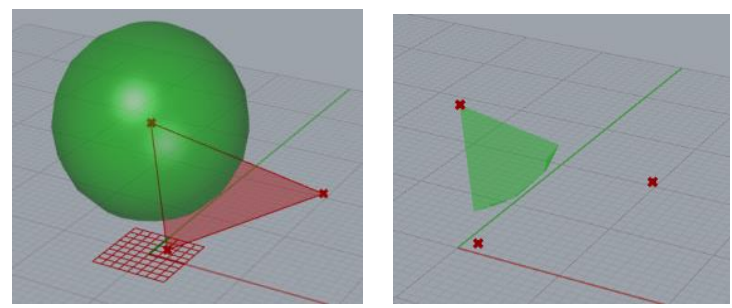

a: Sensor Placed on the Surface b: Resultant area of Coverage

Fig.7. Extraction of intersection area between the surface and the covering spheres with respect to the various positions of the sensor nodes on the surface.

\section{B. Coverage Holes}

The uncovered area was evaluated as the coverage holes on the targeted field i.e. the portion where sensor nodes had no coverage. The total uncovered area was calculated by subtracting the coverage area from the total surface area.

Uncovered Area $=$ Surface Area - Total Coverage Area
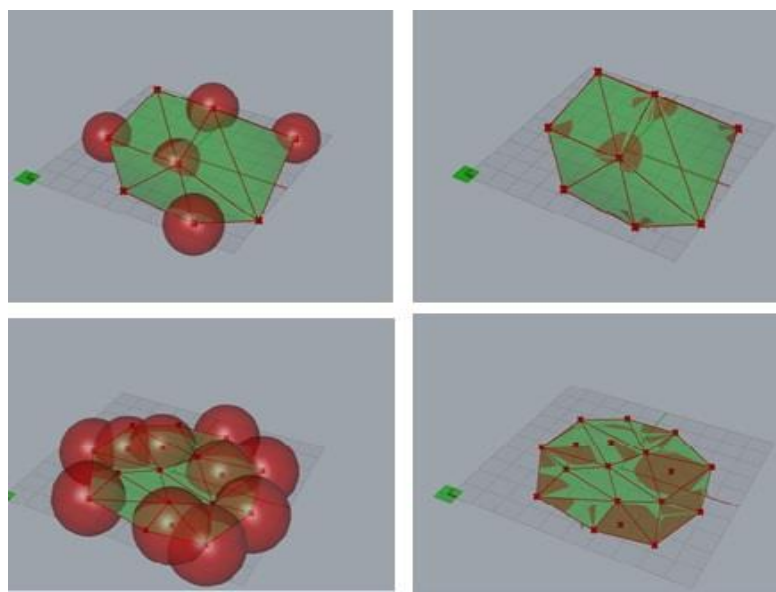

Fig.8. Coverage and coverage holes for the different positions of the sensors on the surface 


\section{Superimpose Area}

In this work, superimpose area was calculated as the covering area more than one sensor nodes with respect to the different number of sensor nodes. The intersection of the sensing spheres on the surface was the superimposed area which was extracted until intersection area among the sensor nodes became null as follows:

If $d<2 *$ r; where $d$ is the distance between the sensor nodes and $r$ is the sensing radius then $\mathrm{S}=$ Sensor Nodes $\cap$ Sensor Nodes Superimpose Area $=\mathrm{S} \cap$ Triangulated Surface Total Superimposing Area $=\sum$ Superimpose Area
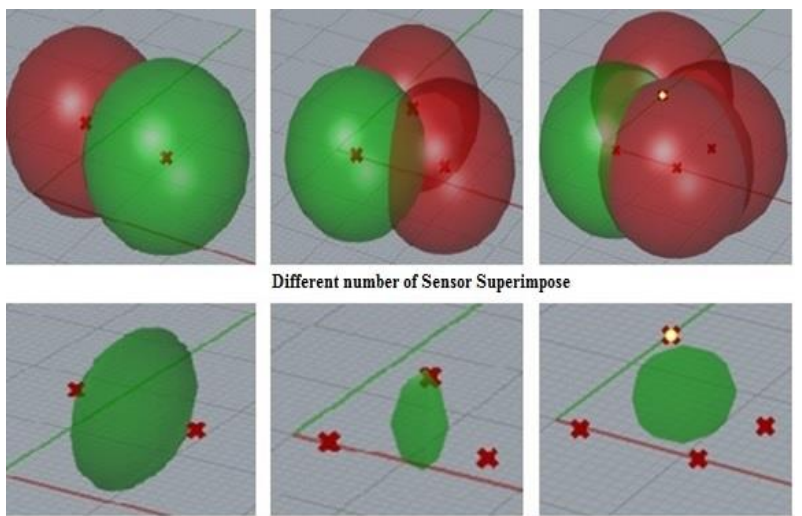

Different number of Sensor Superimpose
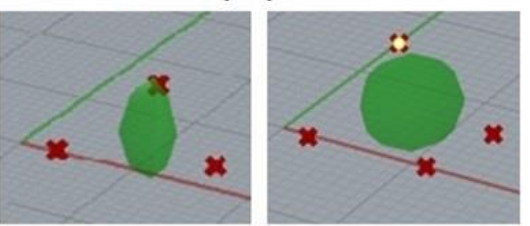

Resultant Volume of Superimpose

Fig.9. Computation of Superimpose volume with respect to a different sensor count
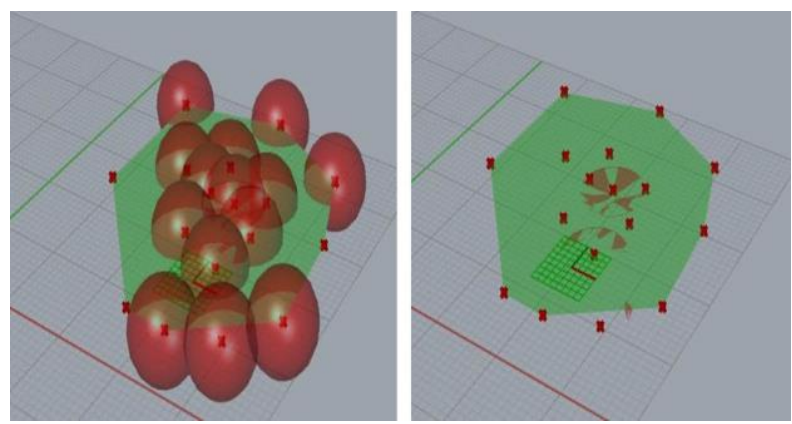

Fig.10. Computation of Superimpose area with respect to superimpose volume on the surface

\section{Performance Evaluation}

The above mention method was computed for different surfaces with the randomly chosen number of surface points and results found as follows:

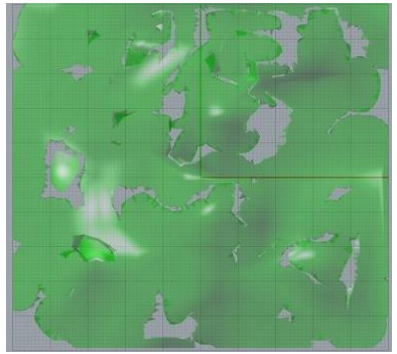

a

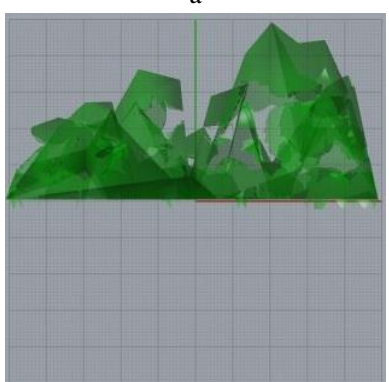

C

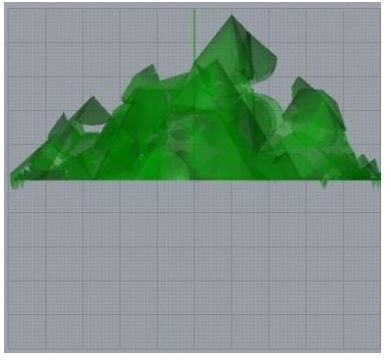

b

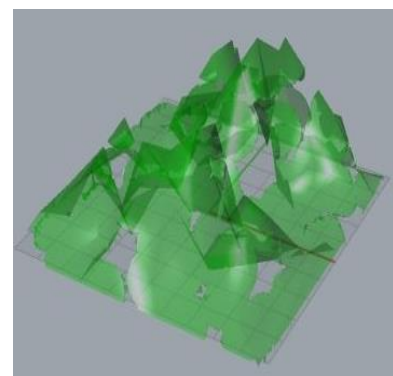

d
Fig.11. Computation for Area Coverage and Uncovered area for different surfaces (a) Top view, (b) Front view, (c) Right view, (d) Perspective view.

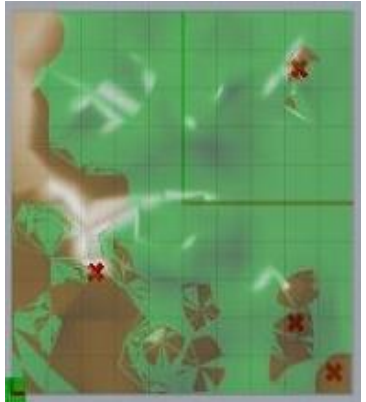

a

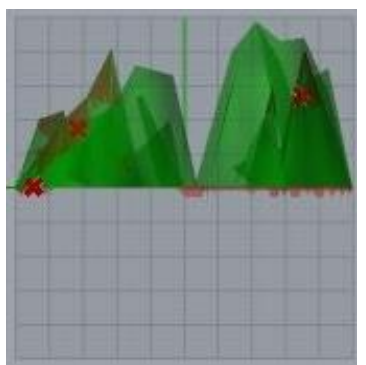

C

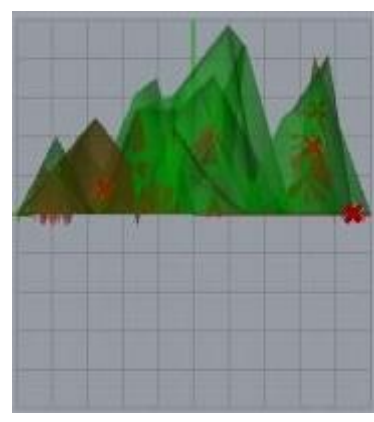

$\mathrm{b}$

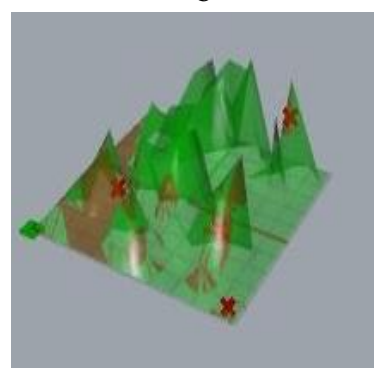

d
Fig.12. Computation for Superimpose area (red) for different surfaces (a) Top view, (b) Front view, (c) Right view, (d) Perspective view. 


\section{RESULTS}

The proposed designed methodology was evaluated using the software MATLAB R2014a. The area coverage and superimpose area and sensor count were calculated for the considered surfaces. A comparison bar graph was prepared for both the deployment methods for four different surfaces and results were presented as follows:

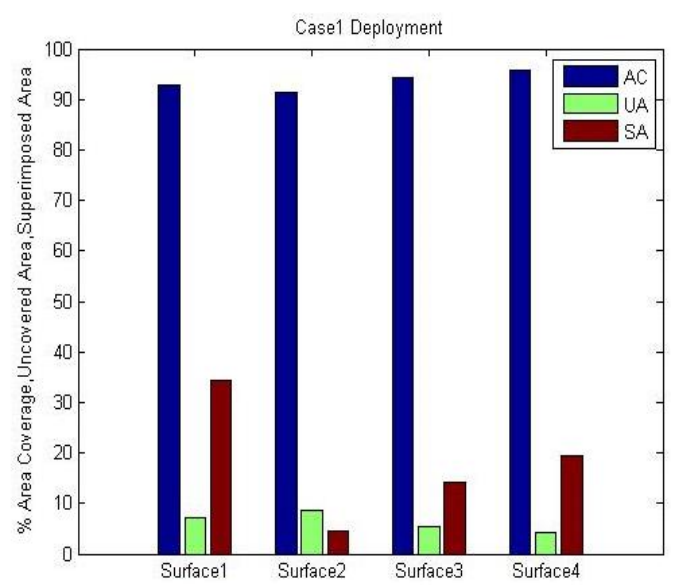

Fig.13. Results obtained for different surfaces using Case1 Sensor deployment

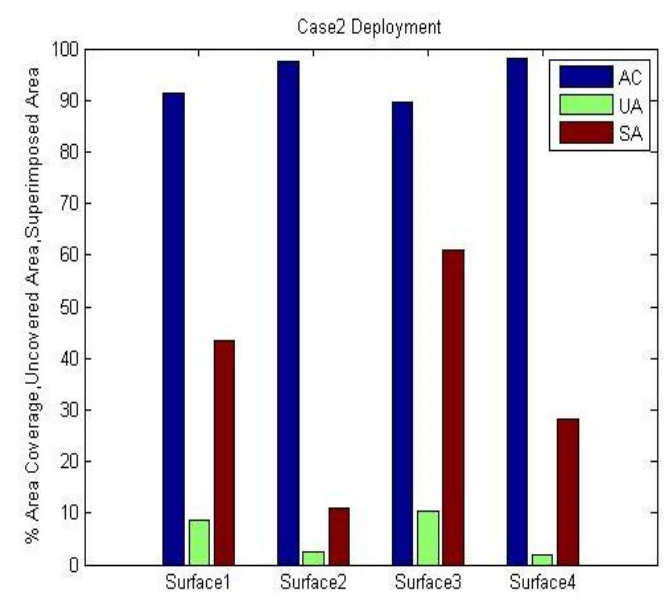

Fig.14. Results obtained for different surfaces using Case2 Sensor deployment

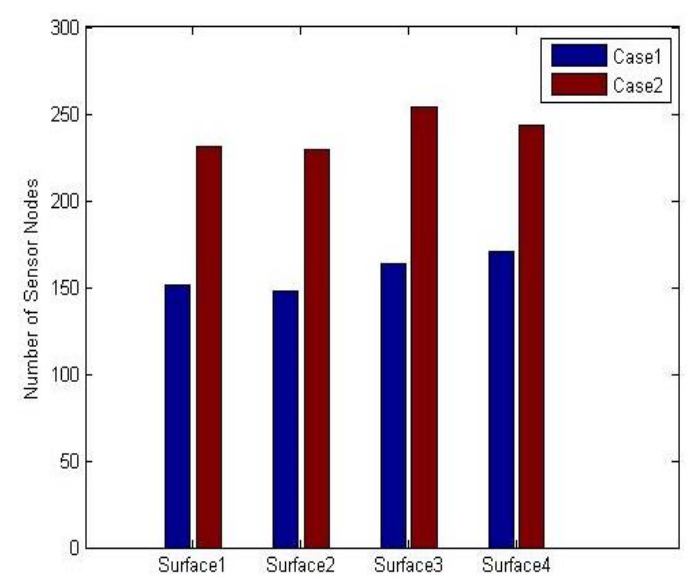

Fig.15. Numbers of sensor count used for different surfaces

\section{CONCLUSION}

In wireless sensor network sensor node localization is a major problem which has a great impact on coverage of the sensor nodes on the targeted field. In this paper, a new node placement strategy is proposed and coverage is computed for different surfaces. Depending on the various surfaces chosen, the overall results found are efficient $92 \%$ to $95 \%$ for both the first method with a superimpose area of $4 \%$ to $34 \%$ and $89 \%$ to $98 \%$ for the second method with a superimpose area of $10 \%$ to $61 \%$ out of total surface area. Observing the results it can be said that the first method gives an efficient result with less sensor count for all the surfaces but the result of the second method varies according to the peaks on the surface with a large number of sensor counts.

The results obtained are promising better geographical coverage with less uncovered area for hilly surfaces. The first method works better for hilly surfaces in comparison to plane surfaces and the second case projection assures full coverage and connectivity for the plane surfaces and which is found to be better than the coverage obtained in ${ }^{[15]}$ considering superimpose area as well and also results obtained for the terrain in ${ }^{[17]}$.

\section{Discussion AND FUture SCOPE}

The clear aim of the proposed deployment strategy is to make the network efficient in terms of coverage and sensor count for Hilly Terrains such that case study of this project will help to estimate the node placement strategy to monitor a surface without going to that original place physically.

A work can be extended until it retrieves a good degree of result. The design strategy for this work can be improved with various node placement methods focusing on a particular degree of coverage area against the number of sensor nodes deployed on the surface. In the same way to make the network efficient in terms of energy consumption one can focus to solve superimpose area for the proposed design topology. For every node placement strategy, the following works can be done to improve the efficiency of the network.

1. Superposition of the sensor nodes can be solved and minimized to get an efficient result in terms of energy consumption.

2. Node deployment methods can be varied to check which method is more efficient: Space-filling polyhedrons can be changed in each method.

3. Sensor Count and sensor Location can be tried to improve in each method to check the connectivity as well.

4. For placement of the sensor nodes obstacles can be considered to improve the work in proper manner.

\section{REFERENCES}

[1] Anvesha Katti, D.K.Lobiyal, "Node Deployment Strategies and Coverage Prediction in 3D Wireless Sensor Network with Scheduling", Advances in Computational 
Science and Technology, ISSN: 0973-6107, Volume 10, November 2017, 2243-2255.

[2] Avantika Thakur, Landran Devendra Prasad, Landran Amit Verma, "Deployment Scheme in Wireless Sensor Network: A Review", International Journal of Computer Applications (0975 - 8887) Volume 163 - No 5, pp-12-15, April 2017.

[3] Chih-Yung Chang, Chao-Tsun Chang, Yu-Chieh Chen, Hsu-Ruey Chang, "Obstacle Resident Deployment Algorithms for Wireless Sensor Network", IEEE, Volume 58, No. 6, Page No. 2925-2941, 2009.

[4] Chinh T. Vu, Yingshu Li, "Delaunay-triangulation based complete coverage in wireless sensor networks", Pervasive Computing and Communication: IEEE International Conference on, ISBN: 978-1-4244-3304-9, Page No. 1-5, Venue: Galveston, TX, USA, 9-13 $3^{\text {th }}$ March 2009.

[5] Chun-Hsien Wu, Kuo-Chuan Lee, Yeh-Ching Chung, "A Delaunay Triangulation based method for wireless sensor network deployment", ISSN: 0140-3664, Volume 30, Issues 14-15, 15 October 2007, Pages 2744-2752.

[6] Gaojuan Fan, Ruchuan Wang, Haiping Huang, Lijuan Sun, Chao Sha, "Coverage Guaranteed Sensor Node Deployment Strategies for Wireless Sensor Network", IEEE, ISSN: 1424-8220, Volume 10, Page No. 2064-2087, 2010.

[7] Guneet Kaur, ParikshitSingla, "Strategy Used to Solve Coverage on Wireless Sensor Network", International Journal of Emerging Research in Management \& Technology, ISSN: 2278-9359, Volume 3, Issue 4, Page No. 187-193, April 2014.

[8] Hassan Chizari, Majid Hosseini, ShukarAbd Razak, Abdul Hanan Abdullah, "Triangle Area Segmentation for Coverage Measurement in Wireless Sensor Network", International Journal of Computer Communications and Network (IJCCN), Volume 1, Issue 1, December 2011.

[9] Hassan Chizari, MajidHosseini, Timothy Poston, ShukarAbd Razak, Abdul Hanan Abdullah, "Delaunay Triangulation as a New Coverage Measurement Method in Wireless Sensor Network", IEEE, ISSN: 1424-8220, Page no. 3163-3176, March 2011.

[10] Haitao Zhang, Cuiping Liu, "A Review on Node Deployment of Wireless Sensor Network", International Journal of Computer Science, ISSN (online): 1694-0814, Issue 6, No. 3, Volume 9, November 2012, 378-383.

[11] Ji Li, L. H. Andrew, Chuan Heng Foh, Moshe Zukerman, Hsiao-Hwa Chen, "Connectivity, Coverage, and Placement in Wireless Sensor Networks", IEEE, ISSN: 1424-8220, Volume 9, pp. 7664-7693, 2009.

[12] Mark De Berg, Otfried Cheong, Marc van Kreveld, Mark Overmars, "Computational Geometry: Algorithms and Applications", Third Edition, Chapter 7, Page no. 147-170 and Chapter 9, Page no. 191-215, Springer Berlin Heidelberg.

[13] Miao Jin, Guodong Rong, Hongyi Wu, Liang Shuai, XiaohuGuo, "Optimal Surface Deployment Problem in Wireless Sensor Networks", 2012 Proceedings IEEE INFOCOM, and ISSN: 4673-0775, Issue 1, Volume 8; Page No. 2345-2353, Venue: Orlando, FL, USA, 25-30 ${ }^{\text {th }}$ March 2012.

[14] Numan Unaldi, SamilTemel, "Wireless Sensor Deployment Method on 3D Environments to Maximize Quality of Coverage and Quality of Network Connectivity", Proceedings of the World Congress Engineering and Computer science, Volume 2, WCECS 2014, ISSN: 2078-0966, 22-24 October 2014, Venue: San Francisco, USA.
[15] Renan Nespolo, Leandro AlvesNeves, Guilherme Roberto, Matheus Ribeiro, "Method Based on Triangulation for Sensor Deployment on 3D Surfaces", IEEE, $29^{\text {th }}$ SIBGRAPI Conference on Graphics, Patterns and Images, ISBN: 9781509035694, Venue: Sao Paulo, Brazil, Volume 1, Pages 464, 4-7 ${ }^{\text {th }}$ October 2016.

[16] Samil Temel, NumanUnaldi, OkyayKaynak, "On Deployment of Wireless Sensors on 3D Terrains to Maximize Sensing Coverage by Utilizing Cat Swarm Optimization with Wavelet Transform", IEEE, ISSN: 2168-2216, Volume 44, No. 1, Page No. 111-120, January 2014.

[17] Sema Oktug, AnarKhailov, HakanTezcan, "3D Coverage Analysis under Heterogeneous Deployment Strategies in Wireless Sensor Networks", the Fourth Advanced International Conference on Telecommunications, IEEE, ISBN: 978-0-7695-3162-5, Page No. 199-204, Venue: Athens, Greece, $8-13^{\text {th }}$ June 2008.

[18] S. Surekha, M. KalyanRam, "Coverage of total Target Field to Form WSN using Delaunay Triangulation", International Journal of Computer Science and Information Technologies (IJCSNT), ISSN: 0975-9646, Volume 4, Issue 6, Page no. 796-799, 2013.

[19] Steven Roman, "Advanced Linear Algebra: Graduate Text in Mathematics", Third Edition, CSpringer 2008.

[20] Usman Mansoor and Habib M. Ammari, "Coverage and Connectivity in 3D Wireless Sensor Networks", Signals and Communication Technology, DOI: 10.1007/978-3642-40066.

[21] Abhik Maiti and Debashish Chakraborty, "Performance analysis of different surface reconstruction algorithms for 3D reconstruction of outdoor objects from their digital images, Springerplus. 2016 Jun 30; 5(1):932. doi: 10.1186/s40064-016-2425-9. eCollection 2016.

[22] Ahmed Abed Alhameed Alkhatib, Gurvinder Singh Baicher, "Wireless Sensor Network Architecture", 2012 International Conference on Computer Networks and Communication Systems (CNCS 2012), IPCSIT, Volume 35, Venue: Kuala Lumpur, Malaysia, 7- $8^{\text {th }}$ April 2012.

[23] A. K. Patil \& A. J. Patil, "ISSUES OF CONNECTIVITY AND COVERAGE IN WIRELESS SENSOR NETWORKS", International Journal of Electrical and Electronics Engineering Research (IJEEER) ,ISSN 2250155X, Vol. 3, Issue 1, pp. 249-258, Mar 2013.

[24] Parveen Kumari, Yudhvir Singh, "Comparative Analysis of Delaunay Triangulation and Square Grid Coverage Strategy for Wireless Sensor Network", International Journal of Advance Research in Computer Science, ISSN: 0976-5697, Volume 2, No. 4, July-August 2011.

[25] Ramnesh Dubey, "Constrained Delaunay Triangulation for Wireless Sensor Network", ISSN: 2278-0661, ISBN: 2278-8727, Volume 8, Issue 4, Page No. 46-53, Jan-Feb 2013.

[26] SANGEETHA S, RAMA LAKSHMI K, “A Survey on Coverage Problems in Wireless Sensor Networks", ISSN: 2278 - 1323, International Journal of Advanced Research in Computer Engineering \& Technology (IJARCET), Vol. 1, Issue 10, December 2012.

[27] Zbigniew H. Nitecky,"Calculus in 3D: Geometry, Vectors and Multivariate Calculus", Tufts University, August 19, 2012. 


\section{Authors' Profiles}

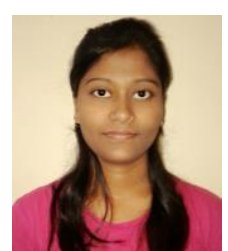

Sunita Saha: Post-graduate student of Computer Science and Engineering from NERIST, Arunachal Pradesh, India.

How to cite this paper: Sunita Saha, "Evaluation of Network Parameters of a Sensor Node Deployment Strategy in Wireless Sensor Network for Hilly Terrains", International Journal of Information Technology and Computer Science(IJITCS), Vol.11, No.1, pp.31-38, 2019. DOI: 10.5815/ijitcs.2019.01.04 